\title{
Nanoscale Vibrational Spectroscopy of Graphene by Large-q EELS
}

Ryosuke Senga $^{1}$, Kazu Suenaga ${ }^{1}$, Shigeyuki Morishita ${ }^{2}$, Paolo Barone ${ }^{3}$, Francesco Mauri ${ }^{4,5}$ and Thomas Pichler $^{6}$

1. Nanomaterials Research Institute, AIST, Tsukuba, Japan.

2. JEOL Ltd., Tokyo, Japan.

3. SPIN-CNR, c/o Università G. D'Annunzio, Chieti, Italy.

4. Dipartimento di Fisica, Università di Roma La Sapienza, Roma, Italy.

5. Graphene Labs, Fondazione Istituto Italiano di Tecnologia, Genova, Italy.

6. Faculty of Physics, University of Vienna, Vienna, Austria.

Since all low-energy quasiparticle excitations are strongly coupled to phonons governing their unique properties, a full description of phonon dispersion is of paramount importance to explore the physical properties of low-dimensional materials. However, existing techniques lack the complementary resolutions in spatial-, energy-, and momentum-space required to measure phonon dispersions in nanoscale. For instance, material phonon dispersions have been usually drawn by inelastic x-ray (neutron) scattering spectroscopy or electron energy loss spectroscopy (EELS) in reflection. Although these techniques have sufficiently high momentum $\left(\sim 0.01 \AA^{-1}\right)$ and energy resolution $(\sim 1 \mathrm{meV})$ to figuratively complete phonon dispersion, measurable objects are basically in the micrometer to millimeter scale with a few hundreds of nanometer thickness. Thus, all local information, such as phonon scatterings at non-perfect structures, is averaged in a spectrum.

Recently, EELS in transmission has emerged as another option to access vibrational charge responses with a scanning transmission electron microscope (STEM) consisting of a monochromatic electron source [1-5]. However, these experiments have been still limited regarding their momentum resolution and to polar materials. Therefore, the full phonon dispersion measurement of atomically thin and apolar materials, such as a graphene monolayer, and the direct evidence of local intensity variations for each vibration mode in nonhomogeneous specimens have not been reported.

Thus, this study demonstrates a nanoscale momentum-resolved phonon spectroscopy on atomically thin and non-polar materials by employing a large-q condition [6]. The experiments were performed by a JEOL TEM (3C2) equipped with a Schottky field emission gun, a double Wien filter monochromator, and delta correctors at $30 \mathrm{keV}$. The momentum-resolved EEL spectra from several layers of graphene and h-BN were first collected (Fig. 1). In this study, it was experimentally found that the inelastically scattered electrons by phonon excitations surprisingly provided sufficient signals detectable even in the third Brillouin zone (as far as $\mathrm{q}>0.75 \AA^{-1}$ ) regardless of the material polarizabilities (Figs. 1c-e). Our extended density functional perturbation theory considering charge modulations fully explained the signal enhancement at a large $\mathrm{q}$ and accurately reproduced the measured spectra including their intensities. This method is also applicable to a freestanding graphene monolayer. Furthermore, the selected phonon maps with distinct vibrational modes were successfully constructed, which unveiled the local variation mode at the graphene edge structures. The probe size used is tens of nanometers to offer a better spatial resolution in few orders of magnitude than any light-source probe measurement. Moreover, it opens up wide possibilities in future nanophysics with its authentic nanoscale phonon spectroscopy with the full dispersive information [7]. 
References:

[1] O Krivanek et al, Nature 514 (2014), p. 209.

[2] MJ Lagos et al., Nature 543 (2017), p. 529.

[3] FS Hage et al., Science Advances. 1 (2018), eaar7495.

[4] C Dwyer et al., Phys. Rev. Lett. 117 (2016), p. 256101.

[5] FS Hage et al., Phys. Rev. Lett. 122 (2019), p. 016103.

[6] R Senga et al., (2019) arXiv:1812.08294

[7] The authors acknowledge funding from JSPS KAKENHI, $17 \mathrm{H} 04797$.

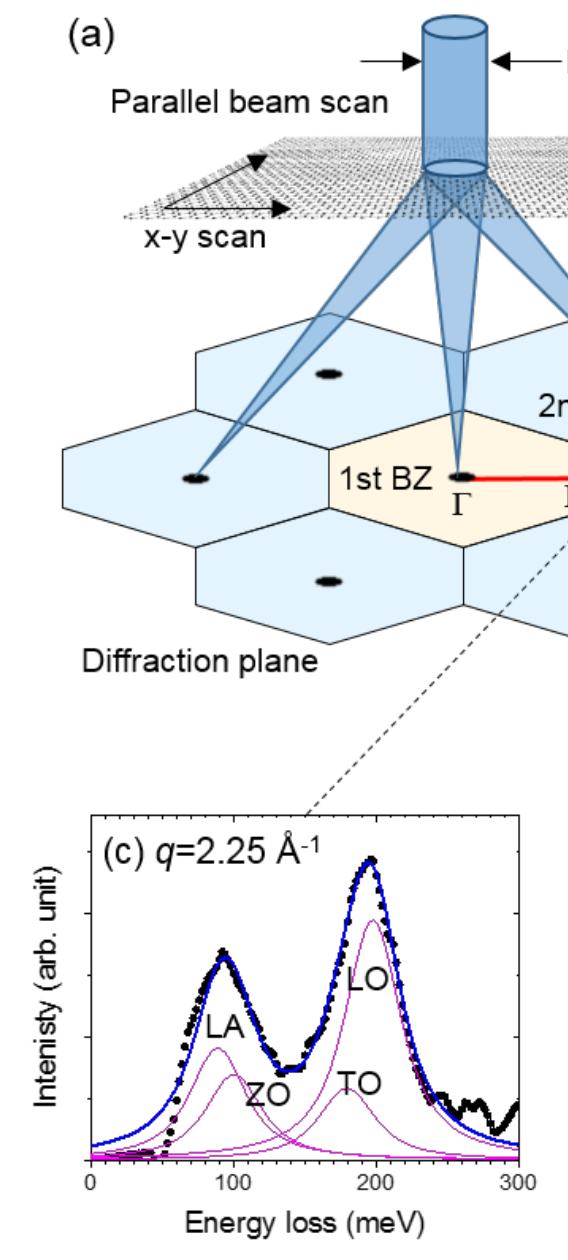

Probe size: 10 40 nm

$\left(\Delta q: 0.02 \sim 0.2 \mathrm{~A}^{-1}\right)$

(b)

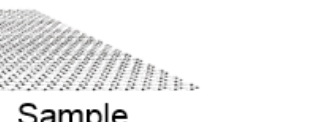

Sample

Integrating momentum

space: $\pm 0.1 \sim 0.2 \mathrm{~A}^{-1}$
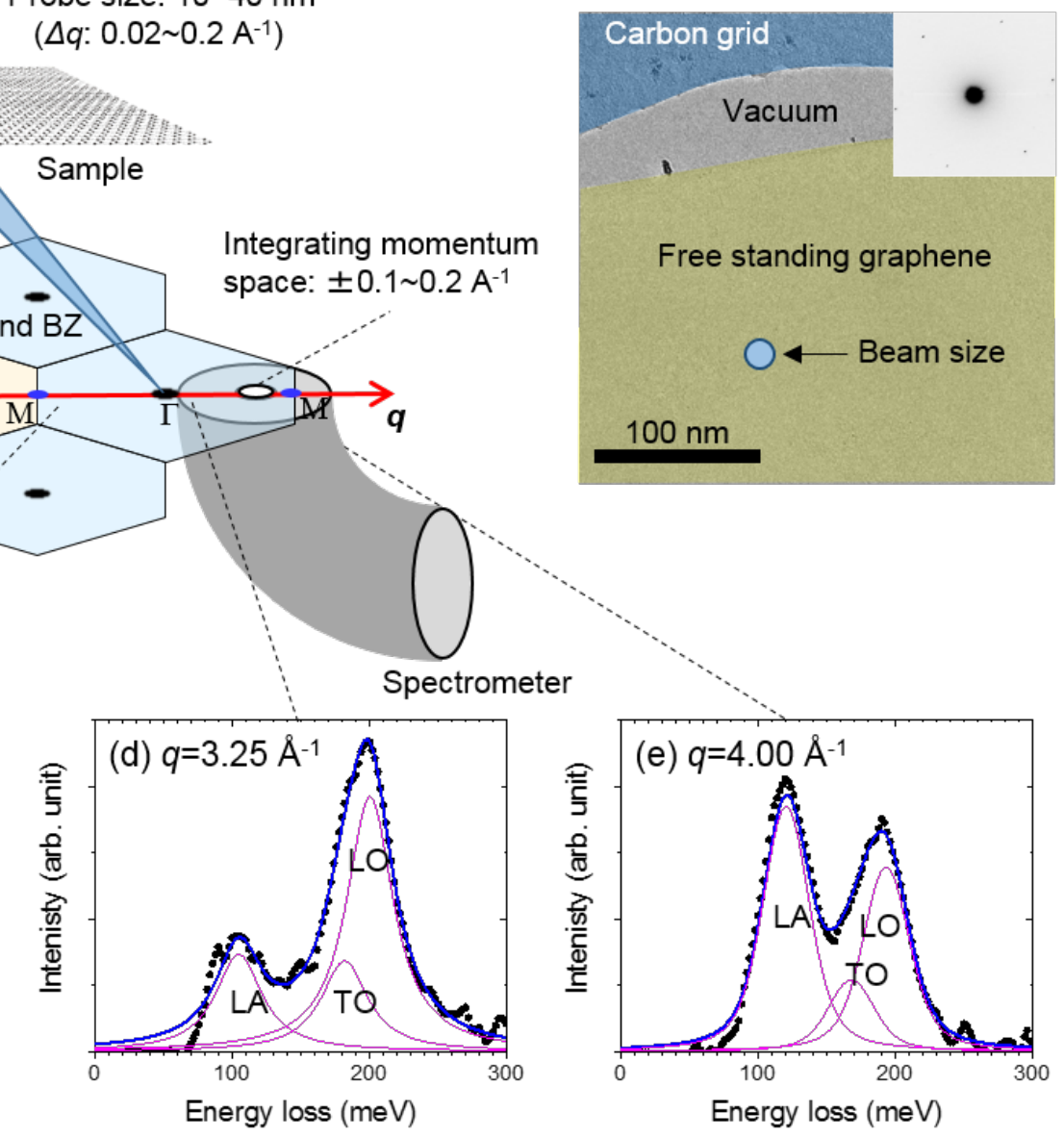

Figure 1. (a) Experimental setup for the momentum-resolved EELS. (b) TEM image of the graphene sample which has several layers and its diffraction pattern (inset). The spectrometer entrance aperture is placed at a given position in the diffraction plane including the first, second, or third Brillouin zones. (ce) Momentum-resolved EEL spectra taken at (c) $\mathrm{q}=2.25$, (d) 3.25 and (e) $4.00 \AA^{-1}$ along the ГМГМ direction as shown in (a). The EEL spectra were collected by STEM mode with the energy resolution set to $30 \mathrm{meV}$ in FWHM. The condition provided the momentum resolution as $\pm 0.1 \AA^{-1}$ and the corresponding probe size as $40 \mathrm{~nm}$. Since the momentum and spatial resolutions balanced each other, the smaller probe size $(\sim 10 \mathrm{~nm})$ is also achievable by integrating the momentum space as $\pm 0.2 \AA^{-1}$. 